\title{
Black Box Warning: Cardiovascular Complications Make Motherhood Unsafe for African American Women
}

\author{
Rolanda Lister ${ }^{1 *}$, Scott Baldwin ${ }^{1}$ and Cornelia Graves ${ }^{2}$ \\ ${ }^{1}$ Vanderbilt University Medical Center, USA \\ ${ }^{2}$ Saint Thomas Midtown Hospital, USA
}

*Corresponding author: Rolanda L Lister, Assistant Professor, Obstetrics and Gynecology, Vanderbilt University Medical Center, $116121^{\text {st }}$ Avenue South, B-1118 Medical Center North, USA.

Received Date: May 20, 2020

Published Date: May 28, 2020

Abstract

Background: In the United States, cardiovascular disease and its complications in pregnancy is the leading killer in mothers. The black maternal mortality rate is quadruple the rate among white women.

Main Body: The reasons for this staggering discrepancy hinge on two central issues: First, black women are more likely to have pre-existing cardiovascular morbidity that increase the risk of maternal mortality. Second, black women are more likely to experience adverse pregnancy outcomes including small for gestational age, gestational diabetes and preeclampsia. These perinatal complications put them at risk for developing long-term cardiovascular disease.

Conclusions: This article is a call to action to recognize that adverse pregnancy outcomes represent an opportunity to intervene in order to change the long-term cardiovascular health of black women.

Keywords: Maternal mortality; Cardiovascular; African American; Blacks; Whites

Abbreviations: : APO (adverse pregnancy outcomes); GDM (Gestational Diabetes Mellitus); PTB (Preterm birth); PEC (Preeclampsia); FGR (Fetal growth restriction); IUFD (Intrauterine Fetal Demise).

\section{Background}

In the United States, maternal mortality is on the rise. In the United States, the causes of maternal death have shifted over time. While the mortality for haemorrhage, infection and hypertensive disorders of pregnancy have decreased from 1987 to 2005, the incidence of mortality due to cardiomyopathy and cardiovascular conditions has increased. Whereby, cardiovascular causes are now the leading cause of maternal mortality in the United States [1]. Adverse pregnancy outcomes, such as preterm birth, gestational diabetes, preeclampsia, placental abruption and fetal growth restriction, are independent risk factors for the development of long-term cardiovascular disease and are overrepresented in black women [2].

Cardiovascular deaths are the leading cause of maternal mortality and black women are disproportionately affected

Cardiovascular deaths are now the leading cause of maternal mortality in the United States [1]. Contrary to trends in other wealthy nations, maternal mortality is on the rise in the United States. Black pregnant women are four times as likely to die as white women. 


\section{Short term effects}

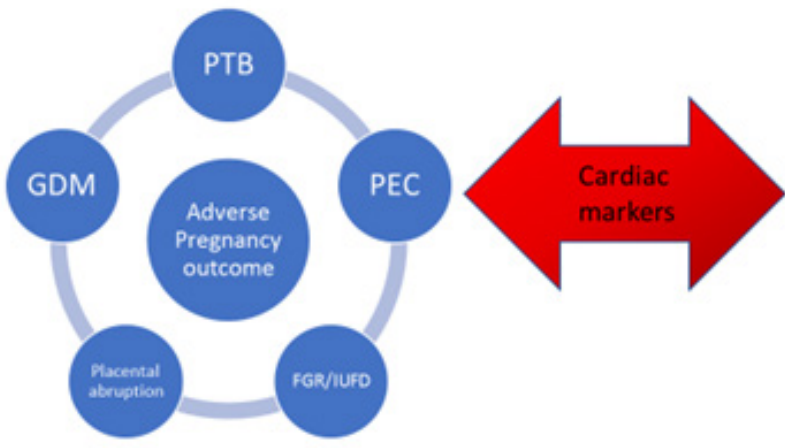

\section{Long-term effects}

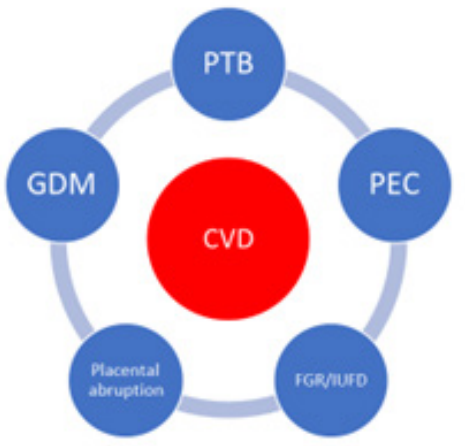

Figure 1: Pregnancy complications in the short term translate to long-term cardiovascular risks.

Pre-existing medical complications such as chronic hypertension and Type 2 diabetes mellitus are more common in obese patients and increase the risk of death. Black women are disproportionately obese and are predisposed to these chronic conditions that often predate pregnancy.

\section{Black women are at higher risk for perinatal complications which lead to the development of cardiovascular disease}

Black women are at higher risk for adverse pregnancy outcomes (APO), which increase the risk of developing cardiovascular disease. APO such as preterm birth, gestational diabetes, preeclampsia, placental abruption, and fetal growth restriction are independent risk factors for the development of long-term cardiovascular disease and are essentially a "stress test". Shown in Figure 1 is the common correlation of cardiac markers in cardiovascular disease sharing similar pathophysiology of perinatal complications.

Women who have delivered preterm have elevated total cholesterol, elevated systolic blood pressure and coronary intimal vessel thickness. Additionally, women who deliver prematurely are more likely to have inflammatory markers such as TNF-alpha and IL-6, both of which are markers for cardiovascular disease [3-5].

Both preeclampsia and coronary artery disease share common biologic markers, including excessive circulation maternal hypertriglyceridemia, free fatty acids, reduced high density lipoprotein, and increased concentration of small LDL and oxidized LDL. Common histopathological features such as foam cells or lipid laden macrophages exist in the spiral arteries of preeclampsia placentas and the vessels of individuals that had fatal coronary artery disease [6].

Gestational diabetes mellitus (GDM) has a higher incidence in minorities, including blacks and Hispanics, versus whites. Over half of women that are diagnosed with gestational diabetes will develop Type 2 diabetes (a cardiovascular risk equivalent) within 10 years of their GDM diagnosis.

Black women are also more likely to have a small for gestational age (SGA) infant, which correlates with an increased risk of both the child and the mother experiencing cardiovascular disease and an increased risk of premature death in the mother.

\section{It's time to act}

Black mothers have an increased risk of dying in the peripartum period and are also at risk of dying in the long term should they develop cardiovascular disease. The United States' disproportionately high black maternal mortality ratio reflects a lack of appreciation for the significance of the pregnancy co-morbidities of black women in this country. Implementing strategies to improve cardiovascular health before and after pregnancy will likely result in a significant reduction in mortality among black women.

\section{Focusing on black maternal cardiac health may reduce perinatal complications}

Cardio-obstetrics is a promising approach that is emerging in many tertiary institutions. This care model uses interdisciplinary teams (Cardiology, Maternal Fetal Medicine, Anaesthesia, Nursing) to care for pregnant patients with heart disease. It focuses on how the pregnancy-associated hemodynamic changes potentially exacerbate cardiac disease. At our institutions, the primary focus of our interdisciplinary team is to reduce cardiac death around the time of labour and delivery and the immediate post-partum period. At Vanderbilt, between October 2016 and August 2019, using a Redcap database, we have used a Cardio-obstetrics model to care for 37 women with significant cardiac disease of which 6/37 self-identified as black. The most common groups of cardiac lesions are valve disease (aortic stenosis, mitral stenosis and pulmonary stenosis) and corrected congenital heart disease (Fontan procedure, arterial switch procedures, and septal closure) accounting for $15 / 37$ or $40 \%$ and $12 / 37$ or $32 \%$ respectively. Other groups of cardiac lesions include coronary artery disease $5 / 37$ or 
$13 \%)$, pulmonary hypertension/heart failure $(3 / 37$ or $8 \%)$ and arrhythmias (2/37 or 5\%). Black women's heart lesions (2/group) were distributed evenly amongst heart failure, repaired congenital heart defects, and valve disease. There was 1 maternal death and no black maternal deaths. Historically, high risk cardiac lesions such as aortic stenosis and mitral stenosis carried a maternal death rate of $10 \%$ and upwards of $25-50 \%$ for pulmonary hypertension. However, this model in our institution has been associated with a maternal mortality of $<3 \%$.

While cardio-obstetrics has shown promise for reducing maternal mortality in pregnant patients with known cardiac disease, it does not consider long-term follow-up care for patients who are at greatest risk of cardiovascular complications (i.e., APO) after their pregnancies is completed. We postulate that similar reductions will be observed with black females identified as being high risk for the development of cardiovascular disease if we use that critical period for prevention and intervention.

\section{Collaboration between Maternal Fetal Medicine Specialists and Cardiologists are essential for long-term follow up for at-risk patients}

The immediate post-partum period is a critical window to establish long-term care with a cardiologist with expertise on management of cardiovascular risk factors. Treatment of an atrisk black patient has the potential to alter the trajectory of her cardiovascular health. It is important for maternal fetal medicine providers to join forces with cardiology national leadership to enhance the education of primary health care providers in recognizing the implications of pregnancy complications for longterm cardiovascular health, particularly among black women. APO should be a routine part of history-taking and should be factored into the cardiovascular risk score.

\section{Conclusion}

Reducing black maternal mortality requires system-level changes, behavioural interventions, and adjustments to clinical care. The cardio-obstetric model has the potential to reduce shortterm mortality in patients with a known history of cardiovascular disease and should be expanded to address the care of women at risk of developing cardiovascular disease long-term. High-risk obstetric providers and cardiologists should join forces leading the way in educating the medical community that APO are associated with cardiovascular complications such as Type 2 Diabetes Mellitus, chronic hypertension, metabolic syndrome and stroke. Pregnant women with elevated cardiovascular risks should have life-long health care.

Similar pathophysiology exists for both clinical entities that lead to adverse perinatal events and cardiovascular events. Abbreviations: GDM (Gestational Diabetes Mellitus), PTB (Preterm birth), PEC (Preeclampsia), FGR (Fetal growth restriction), IUFD (Intrauterine Fetal Demise).

\section{Acknowledgement}

None.

\section{Conflict of Interest}

No conflict of interest.

\section{References}

1. Creanga AA, Berg CJ, Syverson C, Seed K, Bruce FC, et al. (2015) Pregnancy-related mortality in the United States, 2006-2010. Obstet Gynecol 125(1): 5-12.

2. (2018) Notice of Retraction and Replacement: "Health Care Disparity and State-Specific Pregnancy-Related Mortality in the United States, 2005-2014" (Moaddab A, Dildy GA, Brown HL, Bateni ZH, Belfort MA, Sangi-Haghpeykar H, Clark SL). Obstet Gynecol 131(4): 746.

3. Heida KY, Velthuis BK, Oudijk MA, Reitsma JB, Bots ML, et al. (2016) Cardiovascular disease risk in women with a history of spontaneous preterm delivery: A systematic review and meta-analysis. Eur J Prev Cardiol 23(3): 253-263.

4. Moayeri M, Heida KY, Franx A, Spiering W, de Laat MW, et al. (2017) Maternal lipid profile and the relation with spontaneous preterm delivery: a systematic review. Arch Gynecol Obstet 295(2): 313-323.

5. Schaaf JM, Liem SM, Mol BW, Abu-Hanna A, Ravelli AC (2013) Ethnic and racial disparities in the risk of preterm birth: a systematic review and meta-analysis. Am J Perinatol 30(6): 433-450.

6. Staff AC, Dechend R, Pijnenborg R (2010) Learning from the placenta: acute atherosis and vascular remodeling in preeclampsia-novel aspects for atherosclerosis and future cardiovascular health. Hypertension 56(6): 1026-1034. 